LA-UR-99-3229

Approved for public release; distribution is unlimited.

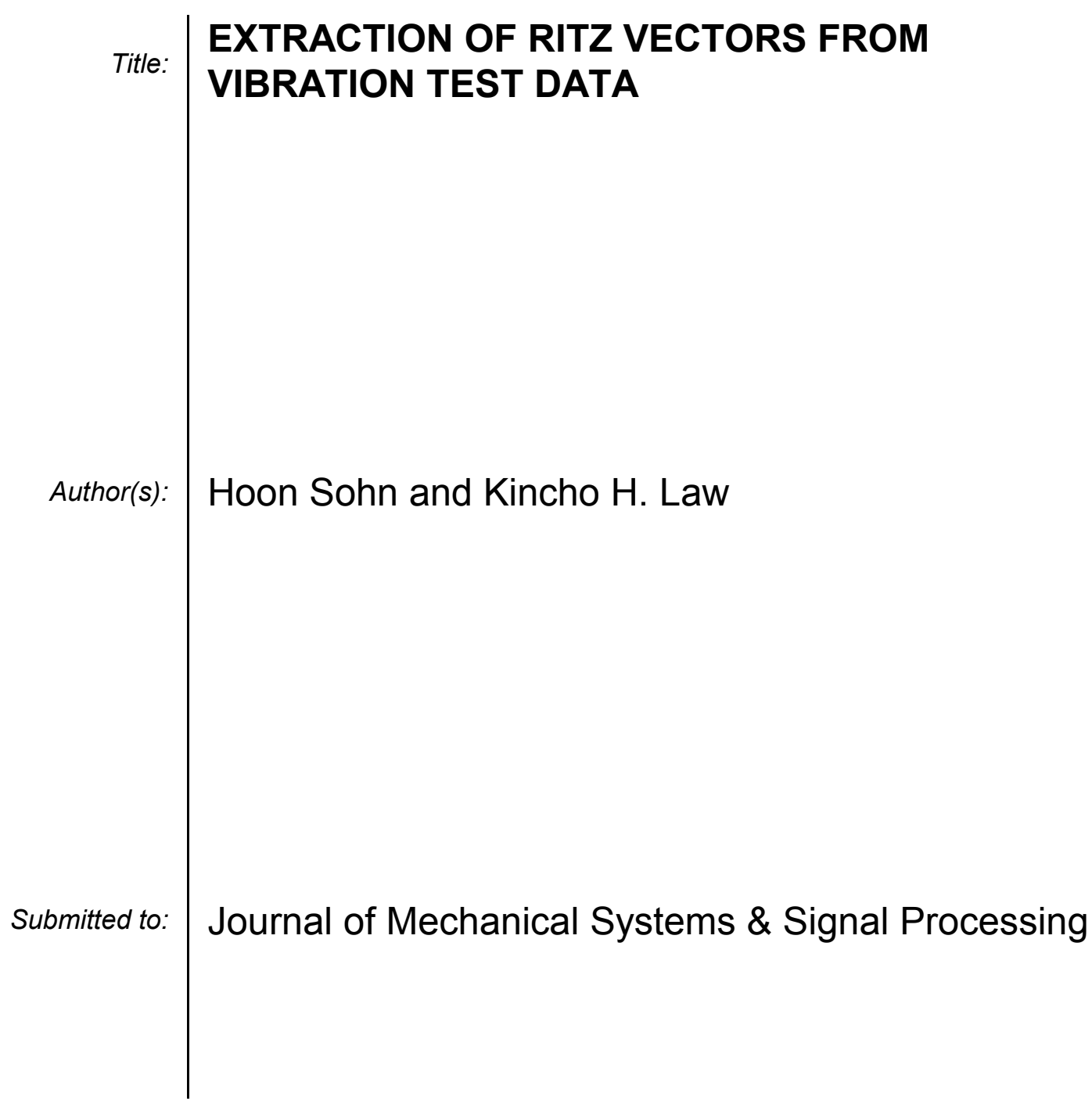

Los Alamos National Laboratory, an affirmative action/equal opportunity employer, is operated by the University of California for the U.S. Department of Energy under contract W-7405-ENG-36. By acceptance of this article, the publisher recognizes that the U.S. Government retains a nonexclusive, royaltyfree license to publish or reproduce the published form of this contribution, or to allow others to do so, for U.S. Government purposes. Los Alamos National Laboratory requests that the publisher identify this article as work performed under the auspices of the U.S. Department of Energy. Los Alamos National Laboratory strongly supports academic freedom and a researcher's right to publish; as an institution, however, the Laboratory does not endorse the viewpoint of a publication or guarantee its technical correctness. 


\title{
EXTRACTION OF RITZ VECTORS FROM VIBRATION TEST DATA
}

\author{
Hoon Sohn \\ Postdoctoral Fellow \\ Engineering Sciences Applications Division \\ Engineering Analysis Group \\ Los Alamos National Laboratory \\ Los Alamos, NM 87545 \\ E-mail: sohn@lanl.gov
}

and

Kincho H. Law

Professor

Department of Civil and Environmental Engineering

Stanford University

Stanford, CA 94305-4020 


\begin{abstract}
Modal parameters obtained from modal testing (such as modal vectors, natural frequencies, and damping ratios) have been used extensively in system identification, finite element model updating, and structural health monitoring. As an alternative to modal vectors, load-dependent Ritz vectors have been shown useful in various areas of structural dynamics such as model reduction and damage detection. The applications of Ritz vectors, however, have been mainly limited in analytical and numerical analyses because of the difficulty to identify them from vibration tests. This paper presents a procedure to extract load-dependent Ritz vectors using a complete flexibility matrix constructed from measured vibration test data. The proposed method can not only construct the Ritz vectors corresponding to the actual load pattern employed in vibration tests, but also generate Ritz vectors from arbitrary load patterns. Experimental data obtained from the vibration test of a grid-type bridge structure are employed to validate and illustrate the proposed extraction procedure.
\end{abstract}




\section{INTRODUCTION}

Modal parameters such as modal vectors, natural frequencies, and damping have been widely employed in many fields of structural dynamics. For example, in numerical dynamic analysis, a multi-degree-of-freedom (MDOF) system can be decoupled into a number of single-degree-offreedom (SDOF) systems using the orthogonality feature of modal vectors and the vibration response of the system can be approximated by the modal superposition of a small set of the SDOF system responses. For vibration test, the response time histories are typically transformed into the frequency domain using a spectral analyzer and the test results are often presented in the form of modal parameters.

It has been shown that load-dependent Ritz vectors have many potential advantages in structural dynamics over modal parameters. For linear dynamic analyses, the response quantities of interest can be approximated more effectively by a smaller number of Ritz vectors than the modal vectors [Nour-Omid and Clough 1984, Wilson et al. 1982]. In numerical analysis, Ritz (or Lanczos) vectors have been used to find partial extremal solutions of large eigenvalue problems [Golub and Van Loan 1996] and to reanalyze a structural system with localized modifications [Carey et al. 1994]. In structural monitoring and damage diagnosis, numerical simulations have shown that Ritz vectors are able to identify damage better than modal vectors [Cao and Zimmerman 1997a, Sohn and Law 2000]. For system identification and damage detection problems, however, the Ritz vectors need to be obtained from experimental test data.

Cao and Zimmerman were probably the first to attempt extracting Ritz vectors from measured vibration data using a state-space formulation [Cao and Zimmerman 1997a]. In this paper, we present a new extraction procedure based on a complete flexibility matrix obtained 
from vibration test data. While the method proposed by Cao and Zimmerman constructs the Ritz vectors corresponding to the actual load pattern imposed on the structure, the proposed method is able to generate Ritz vectors from assumed load patterns as well. The residual flexibility, which is the contribution of unmeasured dynamic modes, is explicitly included in the proposed formulation. The effectiveness of the new extraction procedure is demonstrated using the data obtained from a vibration test of a grid-type bridge structure.

This paper is organized as follows: First, the analytical procedure of generating Ritz vectors is presented. Both the state-space based method and the proposed flexibility matrix based method are then described. We briefly describe the grid-type bridge model employed in the experimental study and the finite element model corresponding to the test structure. The proposed extraction procedure is then demonstrated using the experimental test data. Finally, this paper is concluded with a summary and discussions.

\section{RITZ VECTOR EXTRACTION TECHNIQUES}

In this section, we first review the analytical procedure for the generation of Ritz vectors [Leger et al. 1986]. Second, a state-space based technique is described. A new extraction procedure based on a complete flexibility matrix constructed using measured vibration data is then presented.

\subsection{Analytical Procedure for Generating Ritz Vectors}

Let $\mathbf{M}, \mathbf{C}_{\varsigma}$, and $\mathbf{K}$ denote the mass, damping, and stiffness matrices of a $N$-DOF system, respectively. Then, the equations of motion can be expressed as

$$
\mathbf{M} \ddot{\mathbf{z}}(t)+\mathbf{C}_{\varsigma} \dot{\mathbf{z}}(t)+\mathbf{K} \mathbf{z}(t)=\mathbf{F}(t)
$$


where $\mathbf{z}, \dot{\mathbf{z}}$, and $\ddot{\mathbf{z}}$ are the vectors of displacement, velocity, and acceleration, respectively. $\mathbf{F}(t)$ is a $N \times 1$ vector representing the input force excitation. "Load-dependent" Ritz vectors are generated by taking into account the "spatial distribution" of the dynamic loading which is neglected in the generation of classical modal vectors. Assume that the dynamic loading $\mathbf{F}(t)$ can be separated into a spatial load vector $\mathbf{f}$ and a time function $u(t)$ :

$$
\mathbf{F}(t)=\mathbf{f} u(t)
$$

The first Ritz vector is a static deformation caused by the spatial distribution of the dynamic load vector $\mathbf{f}$ :

$$
\mathbf{K} \overline{\mathbf{r}}_{1}=\mathbf{f}: \text { solve for } \overline{\mathbf{r}}_{1}\left(=\widetilde{\mathbf{r}}_{1}\right)
$$

Then, the first Ritz vector is mass-normalized as:

$$
\mathbf{r}_{1}=\frac{\tilde{\mathbf{r}}_{1}}{\left[\tilde{\mathbf{r}}_{1}^{\mathrm{T}} \mathbf{M} \tilde{\mathbf{r}}_{1}\right]^{1 / 2}}
$$

The subsequent Ritz vectors are recursively generated. Assuming the mass matrix times the previous Ritz vector $\mathbf{M r}_{s-1}$ to be a load, the recurrence relationship computes the next Ritz vector $\overline{\mathbf{r}}_{s}$ :

$$
\mathbf{K} \overline{\mathbf{r}}_{s}=\mathbf{M \mathbf { r } _ { s - 1 }}: \text { solve for } \overline{\mathbf{r}}_{s}
$$

The linear independence of Ritz vectors is achieved using the Gram-Schmidt orthogonalization. That is, the current Ritz vector is mass-orthogonalized with respect to all the previous Ritz vectors:

$$
\tilde{\mathbf{r}}_{s}=\overline{\mathbf{r}}_{s}-\sum_{t=1}^{s-1}\left(\mathbf{r}_{t}^{\mathrm{T}} \mathbf{M} \overline{\mathbf{r}}_{s}\right) \mathbf{r}_{t}
$$

Finally, the current Ritz vector is mass-normalized: 


$$
\mathbf{r}_{s}=\frac{\tilde{\mathbf{r}}_{s}}{\left[\tilde{\mathbf{r}}_{s}^{\mathrm{T}} \mathbf{M} \tilde{\mathbf{r}}_{s}\right]^{1 / 2}}
$$

\subsection{A State-Space Based Method}

Recently, a procedure to extract Ritz vectors based on measured vibration test data has been proposed by Cao and Zimmerman [1997b]. This method involves a minimum rank realization of state-space models from vibration test data. The second order differential equations shown in Equation (1) can be rewritten as the first order differential equations in a number of ways. In classical control theory, the following state-space representation is often used (here, a single number of input is assumed for simplicity):

$$
\dot{\mathbf{x}}=\mathbf{A} \mathbf{x}+\mathbf{B} u
$$

where

$$
\mathbf{A}=\left[\begin{array}{cc}
\mathbf{0} & \mathbf{I} \\
-\mathbf{M}^{-1} \mathbf{K} & -\mathbf{M}^{-1} \mathbf{C}_{\varsigma}
\end{array}\right], \mathbf{B}=\left[\begin{array}{c}
\mathbf{0} \\
\mathbf{M}^{-1} \mathbf{f}
\end{array}\right] \text {, and } \mathbf{x}=\left[\begin{array}{l}
\mathbf{z} \\
\dot{\mathbf{z}}
\end{array}\right]
$$

When the response of a system is measured by the $m$ output quantities $y(t) \quad\left(\in \mathfrak{R}^{m \times 1}\right)$ using sensors such as accelerometer, the measurement vector $y(t)$ is related to the state vectors as follows

$$
\mathbf{y}=\mathbf{C} \mathbf{x}+\mathbf{D} u
$$

where $\mathbf{C}$ is an $m \times 1$ output influence matrix and $\mathbf{D}$ is an $m \times 1$ gain matrix. Note that there exist an infinite number of state-space models that can be constructed from the same input and output relationship.

The state-space based procedure starts with a minimum rank realization of state matrices ( $\hat{\mathbf{A}}, \hat{\mathbf{B}}$, and $\hat{\mathbf{C}}$ ) from the vibration data. In this study, the Eigensystem Realization Algorithm (ERA) is employed for the system realization [Juang 1994]. Note that the realized state matrices 
preserve the actual input-output characteristics regardless of the selection of state variables, $\mathbf{x}$. The state-space based approach for the extraction of experimental Ritz vectors follows closely the analytical procedure for computing the Ritz vectors. The main difference is that the triple state-space matrices $\hat{\mathbf{A}}, \hat{\mathbf{B}}$, and $\hat{\mathbf{C}}$ are employed instead of the system matrices $\mathrm{K}, \mathrm{M}$, and the spatial load vector $\mathbf{f}$.

The static deformation vector $\overline{\mathbf{x}}_{1}$ subject to a load at the excitation point is computed by solving the following equation for $\overline{\mathbf{x}}_{1}$ :

$$
\hat{\mathbf{A}} \overline{\mathbf{x}}_{1}=-\hat{\mathbf{B}}: \quad \text { solve for } \overline{\mathbf{x}}_{1}\left(=\widetilde{\mathbf{x}}_{1}\right)
$$

where the input influence matrix $\hat{\mathbf{B}}$ contains the information of the load vector $\mathbf{f}$. Then, the first Ritz vector is normalized as:

$$
\mathbf{x}_{1}=\frac{\overline{\mathbf{x}}_{1}}{\left[\overline{\mathbf{x}}_{1}^{\mathbf{T}} \overline{\mathbf{x}}_{1}\right]^{1 / 2}}
$$

The subsequent Ritz vectors are recursively generated as follows:

$$
\hat{\mathbf{A}} \overline{\mathbf{x}}_{s}=\mathbf{x}_{s-1}: \text { solve for } \overline{\mathbf{x}}_{s}
$$

The linear independence among Ritz vectors is achieved by orthogonalizing the current Ritz vector with respect to all the previous Ritz vectors:

$$
\widetilde{\mathbf{x}}_{s}=\overline{\mathbf{x}}_{s}-\sum_{t=1}^{s-1}\left(\mathbf{x}_{t}^{\mathrm{T}} \overline{\mathbf{x}}_{s}\right) \mathbf{x}_{t}
$$

Then, the current Ritz vector is normalized:

$$
\mathbf{x}_{s}=\frac{\tilde{\mathbf{x}}_{s}}{\left[\tilde{\mathbf{x}}_{s}^{\mathbf{T}} \tilde{\mathbf{x}}_{s}\right]^{1 / 2}}
$$

Note that $\mathbf{x}_{s}$ is a Ritz vector in the state-space coordinate. Finally, the Ritz vector, which corresponds to the actual measurement points $\mathbf{r}_{s}$, is computed as: 


$$
\mathbf{r}_{s}=\hat{\mathbf{C}} \mathbf{x}_{s}
$$

It should be noted that the normalization and orthogonalization procedures in the state-space method are not identical to those of the analytical generation procedure. Therefore, the Ritz vectors obtained from Equation (16) should be normalized and orthogonalized with respect to the analytical mass matrix $\mathbf{M}$ in order to compare the experimental Ritz vectors with the analytical

ones. In addition, matrix $\hat{\mathbf{A}}$ obtained from vibration tests often contains the "noise" modes as well as the actual system modes. Therefore, the true modes corresponding to the actual system should be first distinguished before the Ritz vector extraction procedure. This process requires some engineering judgment. Various approaches to distinguish the true modes from the noise modes have been described in Juang [1994].

\subsection{A Flexibility Matrix Based Method}

In this section, we present a new extraction procedure of Ritz vectors based on a measured flexibility matrix. A close look at the analytical generation procedure reveals that the generation of Ritz vectors uses the flexibility matrix $\mathbf{G}$ (defined here as the inverse of the stiffness matrix) rather than the stiffness matrix itself. If the modal vectors are mass-normalized such that $\mathbf{V}^{\mathrm{T}} \mathbf{K V}=\mathbf{\Omega}$ and $\mathbf{V}^{\mathrm{T}} \mathbf{M V}=\mathbf{I}$, the flexibility matrix can be represented with the modal parameters [Doebling et al. 1996]:

$$
\mathbf{G}=\mathbf{K}^{-1}=\mathbf{V} \boldsymbol{\Omega}^{-1} \mathbf{V}^{\mathrm{T}}
$$

where $\boldsymbol{\Omega}$ is the diagonal eigenvalue matrix and $\mathbf{V}$ is the corresponding eigenvector (modal vector) matrix.

In most experimental modal analyses, only a few lower modal frequencies and modal vectors are identified. For this case, the flexibility matrix is divided into the modal flexibility, 
which is formed from the estimated frequencies and modal vectors, and the residual flexibility formed from the residual modes:

$$
\mathbf{G}=\mathbf{G}_{m}+\mathbf{G}_{r}=\mathbf{V}_{m} \Omega_{m}^{-1} \mathbf{V}_{m}^{\mathrm{T}}+\mathbf{V}_{r} \Omega_{r}^{-1} \mathbf{V}_{r}^{\mathrm{T}}
$$

where the subscripts $m$ and $r$ denote the measured and residual quantities, respectively. Here, the modal flexibility $\mathbf{G}_{m}$ is easily constructed using the measured natural frequencies and modal vectors. The residual flexibility $\mathbf{G}_{r}$ is the contribution of the unmeasured dynamic modes to the full flexibility matrix. In general, the contribution of lower modes, which are normally estimated in experimental modal analyses, is more significant than those of higher modes because the contribution of each mode is inversely proportional to the magnitude of the corresponding natural frequencies. Deobling et al. [1996] report that the contribution of the residual flexibility matrix is generally about $3-10 \%$ of the complete flexibility matrix. However, as shown in later examples, the contribution of the residual flexibility cannot be neglected in the extraction procedure of Ritz vectors.

The residual flexibility is constructed using a residual function $\mathbf{R}(\omega)$, which is computed by subtracting the reconstructed response of the identified modes from the measured frequency response functions (FRFs) $\mathbf{H}(\omega)$ :

$$
\mathbf{R}(\omega)=\mathbf{H}(\omega)+\omega^{2} \mathbf{V}_{m}\left(\boldsymbol{\Omega}_{m}-\omega^{2} \mathbf{I}\right)^{-1} \mathbf{V}_{m}^{\mathrm{T}}=-\mathbf{G}_{r} \omega^{2}
$$

Curve-fitting $\mathbf{R}(\omega)$ over a set of frequency samples $\left(\omega \in \omega_{\text {set }}\right)$ yields an estimate of the residual flexibility:

$$
\mathbf{G}_{r}=-\sum_{\omega \in \omega_{s e t}} \frac{\mathbf{R}(\omega)}{\omega^{2}}
$$

In this paper, $\mathbf{H}(\omega)$ and $\mathbf{R}(\omega)$ are assumed to be squared matrices. That is, individual modal testing is conducted by applying an impulse excitation force to a single degree of freedom (DOF) 
and the excitation is repeated for all measured DOFs. Doebling et al. [1996] discuss the extraction of a complete flexibility when the number of excitation points are less than that of the response points. While damping is not included in the formulation of the residual flexibility, Equation (19) can be easily modified to take into account the damping.

From the measured flexibility matrix $\mathbf{G}\left(=\mathbf{G}_{m}+\mathbf{G}_{r}\right)$ and the analytical mass matrix $\mathbf{M}$, the first Ritz vector can be computed as:

$$
\overline{\mathbf{r}}_{1}=\mathbf{G f}
$$

where $\mathbf{f}$ is the spatial load distribution vector defined in Equation (2). The first Ritz vector is, then, mass-normalized as:

$$
\mathbf{r}_{1}=\frac{\tilde{\mathbf{r}}_{1}}{\left[\tilde{\mathbf{r}}_{1}^{\mathrm{T}} \mathbf{M} \tilde{\mathbf{r}}_{1}\right]^{1 / 2}}
$$

The subsequent Ritz vectors are recursively generated in a similar fashion to Equation (5). The only difference is that the flexibility matrix is directly constructed from the test data instead of inverting the analytical stiffness matrix. The orthogonalization and mass-normalization procedures are identical to Equations (6) and (7).

It is worthwhile to compare the flexibility based extraction procedure with the state-space based procedure. In the state-space method, the input influence matrix $\hat{\mathbf{B}}$ in Equation (11) is estimated using the information of the experimentally applied load. While the state-space based method only identifies Ritz vectors corresponding to the specific excitation pattern used in the actual modal testing, the flexibility based method is able to generate Ritz vectors with arbitrary load patterns as well. That is, the spatial load distribution vector $\mathbf{f}$ in Equation (21) can be assigned arbitrarily to generate different sets of Ritz vectors. Furthermore, the contribution of the residual flexibility is not included in the state-space based method. (The influence of the residual flexibility on the accuracy of the estimated Ritz vectors will be addressed in Section 5.) Note that 
both methods require an appropriate approximation for the mass matrix. However, for problems that stiffness changes are the main concern as in the case for damage detection, the exact estimation of the mass matrix is not necessarily an important issue. One will be more likely interested in the change of Ritz vectors caused by stiffness perturbation. It should also be pointed out that Alvin [1994] has presented a theory to transform the realized state-space models into the corresponding second-order structural parameters such as stiffness, mass, and damping matrices based on an objective normalization of physical-coordinated modal vectors. Using these directly computed stiffness and mass matrices, an arbitrary set of Ritz vectors could also be extracted in theory.

\section{AN EXPERIMENTAL BRIDGE MODEL}

For this study, a grid-type bridge model has been constructed and tested at the Hyundai Institute of Construction Technology (HICT), Korea (Figure 1). The steel bridge model consists of two parallel girders and six evenly spaced cross beams connecting the two girders. The girders are steel rectangular tubes and the cross beams are C-shape members. Using impact excitations, we extract Ritz and modal vectors from the vibration response of this test structure.

A SA-390 signal analyzer with four channels is used for the analog to digital conversion of accelerometer signals and the Fast Fourier Transform (FFT) calculation. Data acquisition parameters are specified such that a frequency response function (FRF) in the range of 0 to 100

$\mathrm{Hz}$ could be estimated. Each spectrum is computed by averaging three 8-second long time histories. A total of 2048 points are sampled for a 8-second time period and this sampling rate produces a frequency resolution of $0.125 \mathrm{~Hz}$. An exponential window is applied to all measured time histories prior to the FFT calculation. 
For measurements, a Dytran 5801A4 impact hammer and three Dytran 3100B accelerometers with a normal sensitivity of $100 \mathrm{mV} / \mathrm{g}$ are used. The excitation is applied at each node as shown in Figure 2. The sensors measure the vertical accelerations at the twelve nodes as indicated in Figure 2. Note that since the SA-390 data acquisition system has only four channels and there are three accelerometers, the first channel is always connected to the input hammer and the remaining three channels are connected to three accelerometers. To complete one set of modal test, the hammer excitation is repeated twelve times at one point and the three accelerometers are moved from one set of three nodes to another set of three nodes after every three excitations. Note that each FRF is computed by averaging the three response time histories, and there are twelve measurement points and three accelerometers.

The rational polynomial technique is employed to extract the first six natural frequencies and the corresponding modal vectors from the recorded FRFs [Richardson and Formenti 1982]. Figure 3 shows a typical FRF and coherence function of the test structure. Figure 3 (a) is a FRF obtained at node 5 when the structure is excited at node 4 . Figure 3 (b) presents the corresponding coherence function. Figure 3 (b) shows that the value of the coherence function is close to unity at the resonance frequencies, which indicates that the output signals from the sensors are reasonably strong as compared to the noise levels.

To validate the linearity assumption of the structure response, a reciprocity check has been conducted. Figure 4 compares the FRFs obtained at node 3 with an input at node 14 and at node 14 with an excitation at node 3 . For an ideal linear structure, these two FRFs should be identical. For the grid structure, the linearity assumption seems valid up to the fourth mode but the difference between the FRFs becomes noticeable after $40 \mathrm{~Hz}$. 


\section{ANALYTICAL MODELING OF THE TEST STRUCTURE}

A finite element (FE) model for the grid type bridge structure is constructed using twenty threedimensional beam elements. As shown in Figure 2, a girder segment between two nodes or a cross beam is modeled as a single element. An elastic modulus of $2.0 \times 10^{5} \mathrm{MPa}$, a mass density of $7850 \mathrm{~kg} / \mathrm{m}^{3}$, and a Poission ratio of 0.2 are specified for the model. Since the accelerometers measure only the vertical movement of the structure, the lateral DOFs are not included in the analytical model. Therefore, each node of an element has two translational DOFs and three rotational DOFs. The model has a total of 64 DOFs including four rotational DOFs at the boundary. Both ends of the beam are modeled as simple pinned connections.

A pinned connection is modeled by a ball bearing with a $35 \mathrm{~mm}$ diameter in the experimental setup. Based on a preliminary vibration test, the boundary conditions appear to be less accurately modeled. The boundary conditions are then modified by introducing rotational springs at the rotational DOFs of the boundaries. Furthermore, additional springs are added to the rotational DOFs at both ends of the cross beams to simulate the bolted connection between the girders and the cross beams. After these modifications, the relative errors of the first six natural frequencies between the analytical model and the test structure fall within $4 \%$.

Table 1 compares the natural frequencies of the analytical model computed after model updating with the experimental frequencies. Here, the experimental frequency $\hat{\omega}$ is a mean value of the twelve frequencies estimated with an impact load applied at nodes 2-7, and 10-15, respectively. The damping ratios for the first six modes are also estimated: $0.8725 \%, 0.9780 \%$, $0.3420 \%, 0.6151 \%, 0.4231 \%$, and $0.4981 \%$.

Figure 5 displays the analytical and experimental modal vectors of the first six modes. All figures are plotted in the global X-Y plane of Figure 2, viewing the structure from the side. As 
for the scaling of the modal or Ritz vectors, a mass-normalization is conducted. However, since the DOFs of the analytical model do not coincide with the DOFs of the experimental modal vectors, a reduced analytical mass matrix is first computed using the Guyan condensation procedure. Both the analytical and experimental vectors are normalized with respect to the reduced mass matrix. Errors arisen from the model reduction are found to be minimum since the inertial forces associated with the omitted rotational and axial DOFs (slave DOFs) are negligible in this example.

\section{EXPERIMENTAL VERIFICATION}

The new extraction procedure for Ritz vectors is demonstrated using the vibration data obtained from the test structure. Special focus is placed on the influence of the residual flexibility on the accuracy of extracted Ritz vectors. Figure 6 compares the first six Ritz vectors estimated by the flexibility based method with the corresponding Ritz vectors computed from the FE model. Imaginary point loads are simultaneously applied to nodes 2 and 13 (upward point load at node 2 and downward point load at node 13), and the corresponding Ritz vectors are generated. A complete flexibility matrix, including the modal and residual flexibility matrices estimated from the measured FRFs, is employed for the extraction of Ritz vectors. Figure 6 shows a good agreement between the analytical and experimental Ritz vectors. The first Ritz vector is equivalent to a static deflection pattern observed when the unit loads are applied at nodes 2 and 13. Note that the discrepancy between the analytical and experimental Ritz vectors increases for higher Ritz vectors.

To investigate the influence of the residual flexibility, Figure 7 shows the experimental Ritz vectors obtained by only using the measured modal flexibility. Comparison of Figure 6 and 
Figure 7 reveals that the inclusion of the residual term significantly improves the accuracy of higher Ritz vectors. More quantitative analysis is presented in Table 2, where the Modal Assurance Criterion (MAC) value is defined as follows:

$$
\operatorname{MAC}(i, j)=\frac{\left(\mathbf{r}_{i}^{\mathrm{T}} \mathbf{M} \hat{\mathbf{r}}_{j}\right)^{2}}{\left(\mathbf{r}_{i}^{\mathrm{T}} \mathbf{M} \mathbf{r}_{i}\right)\left(\hat{\mathbf{r}}_{j}^{\mathrm{T}} \mathbf{M} \hat{\mathbf{r}}_{j}\right)}
$$

where $\mathbf{r}_{i}$ and $\hat{\mathbf{r}}_{j}$ are the analytical and experimental Ritz vectors, respectively.

Although not presented here, the state-space based method basically produces the same results as the proposed method with only using the modal flexibility. Furthermore, as mentioned earlier, only the flexibility based method allows generating Ritz vectors from any fictitious load patterns as well as the actual load pattern applied during the tests.

In Table 3, the experimental Ritz vectors corresponding to a point load at nodes 2 - 7 and 10 - 15 are extracted, and the comparison of MAC values with the corresponding analytical Ritz vectors are presented. Again, the measured complete flexibility is used for all cases in the table. For brevity, only the diagonal components of the MAC values are shown in the table. The result indicates that the Ritz vectors can be successfully generated from all the load patterns imposed.

Next, the Ritz vectors are estimated using only the modal flexibility, and the MAC values are presented in Table 4. For all cases, the MAC values between the analytical and experimental Ritz vectors fall below 0.8 after the fourth Ritz vectors. The inclusion of the residual flexibility matrix into the extraction procedure allows the contribution of higher residual modes into experimentally estimated Ritz vectors significantly improving the accuracy of the estimated Ritz vectors. 


\section{SUMMARY AND DISCUSSIONS}

In this paper, a new procedure has been proposed to extract load-dependent Ritz vectors using a statically complete flexibility matrix. First, modal flexibility and residual flexibility matrices are estimated from measured FRFs. Then, Ritz vectors are recursively generated using the flexibility matrix constructed.

The procedure is successfully demonstrated using an experiment of a grid-type bridge structure. Particularly, the influence of the residual flexibility on the extracted Ritz vectors is investigated. The proposed method has at least two advantages over the state-space method: (1) the inclusion of the residual flexibility significantly improves the accuracy of the estimated Ritz vectors, and (2) the proposed method is able to generate Ritz vectors from any arbitrary load patterns. The increased amount of information and better sensitivity to structural parameter changes, which are achievable by multiple loading and careful selection of load patterns, could improve the results of damage detection, test-analysis correlation, model refinement, and system identification.

Using the Ritz vectors extracted with the procedure described here, Sohn and Law (1999) have applied and analyzed the various damage cases introduced to the grid-type bridge structure

presented in this study. Damage diagnoses of the grid-type bridge structure indicates that the employment of Ritz vectors provides better indication of the actual damage locations than using the modal vectors. The reason is that, using appropriate load patterns, Ritz vectors can be made more sensitive to damage than modal vectors. The superior performance of Ritz vectors over modal vectors attributes to (1) the better sensitivity of Ritz vectors over modal vectors and (2) the increase amount of information obtained by employing multiple load patterns. 
There have been continuing interest in the application of Ritz vectors for experimental dynamics problems. Rhee (2000) shows how Ritz vectors can be used to effectively characterize the changes of the system dynamic properties caused by localized non-proportional damping effects and/or structural perturbation. Model reduction and mode shape expansion techniques are further developed for model updating purpose based on experimentally extracted Ritz vectors. These techniques are demonstrated using experimental data obtained from an eight DOF massspring system. To verify the feasibility and accuracy of the experimental Ritz vectors, Zimmerman (1999) utilized the dynamic and static test data obtained from a space shuttle vertical stabilizer. Compared to static test data, accurate Ritz vectors were extracted from a modal survey test using both accelerometers and a laser scanning vibrometer. Then, a matrix update approach using the measured Ritz vectors is conducted to update an FE model and to

estimate the location and extent of damage. The proposed Ritz vector extraction procedure described in this paper could find many applications in system identification and damage detection problems.

\section{ACKNOWLEDGMENTS}

This work is supported by National Science Foundation under Grant No. CMS-95261-2. The authors wish to recognize the Hyundai Institute of Construction Technology (HICT), Korea for providing the laboratories and their personnel. The authors also would like to thank Mr. Jeong Hwan Jang of Seoul National University for his contributions to the experimental portion of this paper. 


\section{REFERENCE}

1. B. Nour-Omid and R.W. Clough 1984 Earthquake Engineering and Structural Dynamics 12, 565-577. Dynamics analysis of structures using Lanczos coordinates.

2. E.L. Wilson, M.W. Yuan and J.M. Dicken 1982 Earthquake Engineering and Structural Dynamics 10, 813-821. Dynamic analysis by direct superposition of Ritz vectors.

3. G.H. Golub and C.F. Van Loan 1996 Matrix Computations, The Johns Hopkins University Press, Maryland.

4. C.M.M. Carey, G.H. Colub and K.H. Law 1994 International Journal for Numerical Methods in Engineering 37, 2857-2883. Lanczos-based method for structural dynamic reanalysis problems.

5. T.T. Cao and D.C. Zimmerman 1997a Proceedings of the 15th International Modal Analysis Conference, Orlando, FL, 1319-1324. Application of load-dependent Ritz vectors in structural damage detection.

6. H. Sohn and K.H. Law 2000 Probabilistic Engineering Mechanics 15, 139-153. Application of load-dependent Ritz vectors to Bayesian probabilistic damage detection.

7. T.T. Cao and D.C. Zimmerman 1997b Proceedings of the 15th International Modal Analysis Conference, Orlando, FL, 1036-1042. A procedure to extract Ritz vectors from dynamic testing data.

8. P. Leger, E.L. Wilson and R.W. Clough 1986 Technical Report UCB/EERC-86/04, Earthquake Engineering Research Center, University of California Berkeley, Berkeley, CA. The use of load-dependent Ritz vectors for dynamic and earthquake analyses.

9. J.N. Juang 1994 Applied System Identification, Prentice Hall, Englewood Cliffs, New Jersey. 
10. S.W. Doebling, L.D. Peterson and K.F. Alvin 1996 American Institute of Aeronautics and Astronautics 34,1678-1685. Estimation of reciprocal residual flexibility from experimental modal data.

11. K.F. Alvin 1994 American Institute of Aeronautics and Astronautics 32, 397-406. A secondorder structural identification procedure via state-space based system identification.

12. M.H. Richardson and D.L. Formenti 1982 Proceedings of the 1st International Modal Analysis Conference, Orlando, FL, 167-181. Parameter estimation form frequency response measurements using rational fraction polynomials.

13. H. Sohn and K.H. Law 1999 Submitted for publication of Journal of Engineering Mechanics, ASCE. Damage diagnosis using experimental Ritz vectors.

14. W. Rhee 2000 Linear and nonlinear model reduction in structural dynamics with application to model updating. Ph.D. Dissertation, Texas Tech University, Lubbock, TX.

15. D.C. Zimmerman 1999 Key Engineering Materials 167-168, 76-90. Looking into the crystal ball: the continued need for multiple viewpoints in damage detection. 


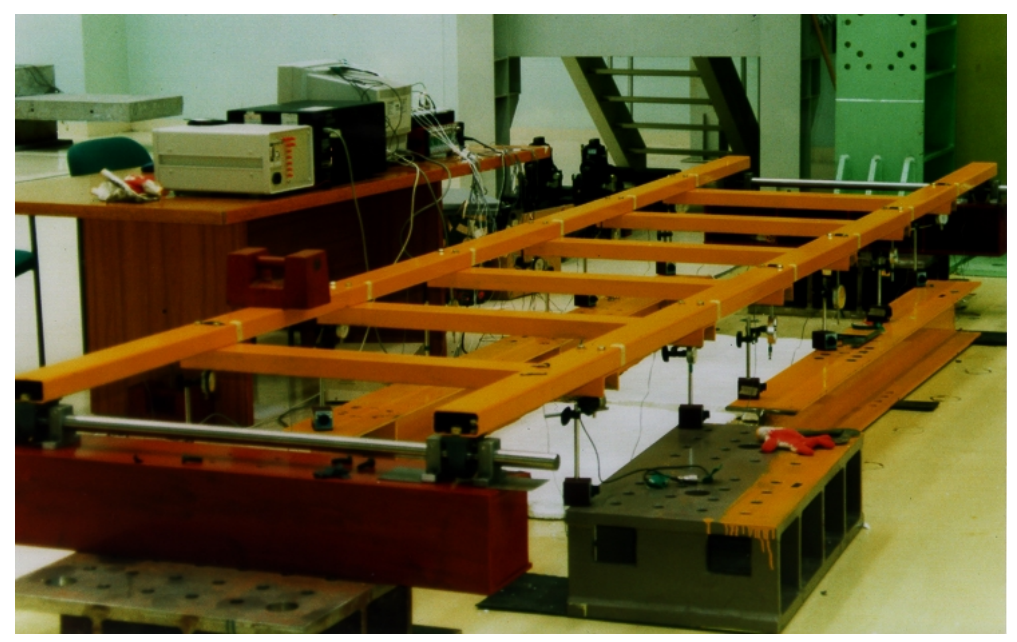

Figure 1: An overview of a grid-type bridge structure 


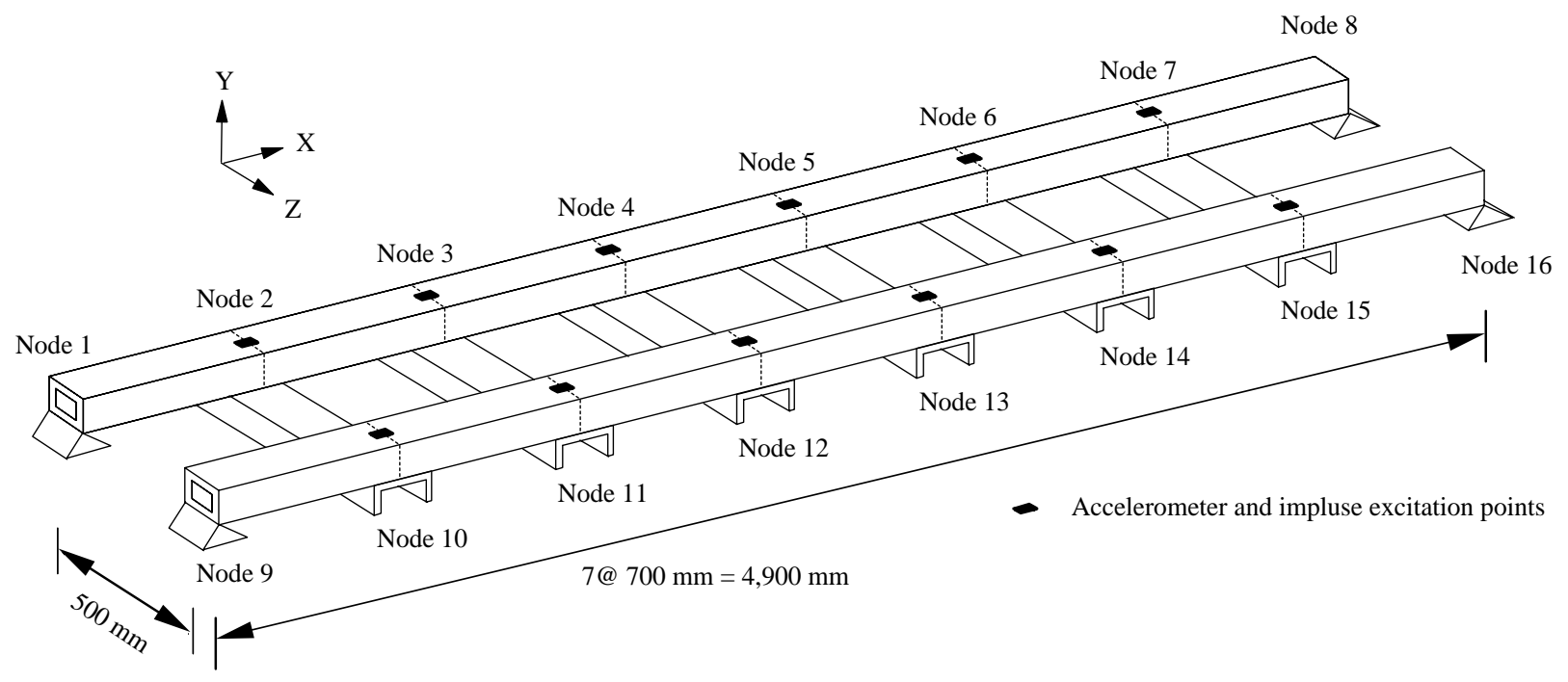

Figure 2: Configuration of a grid-type bridge model 

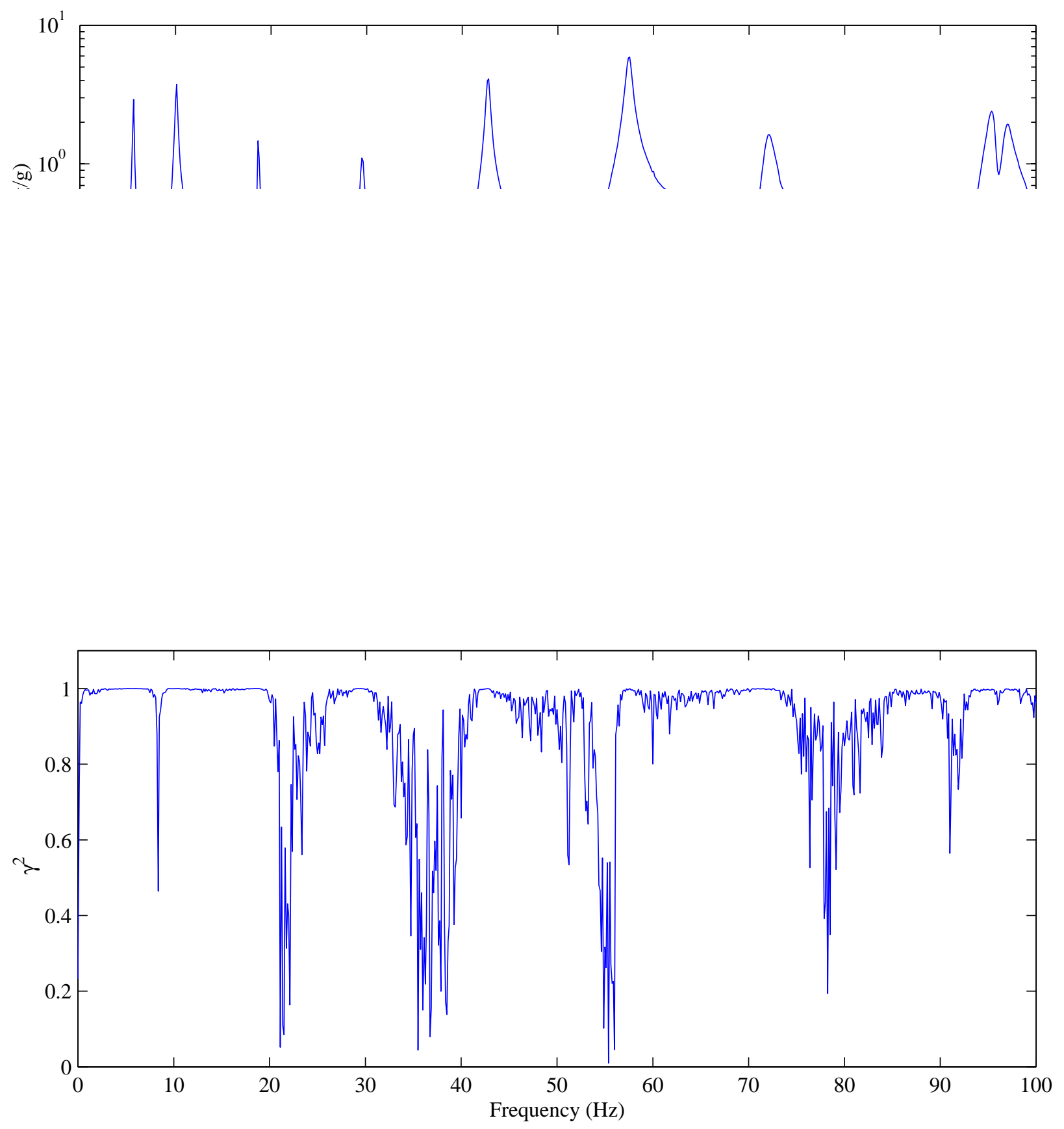

(b) A coherence function of node 5 with node 4 input

Figure 3: A typical FRF \& coherence function of the grid-type bridge model 


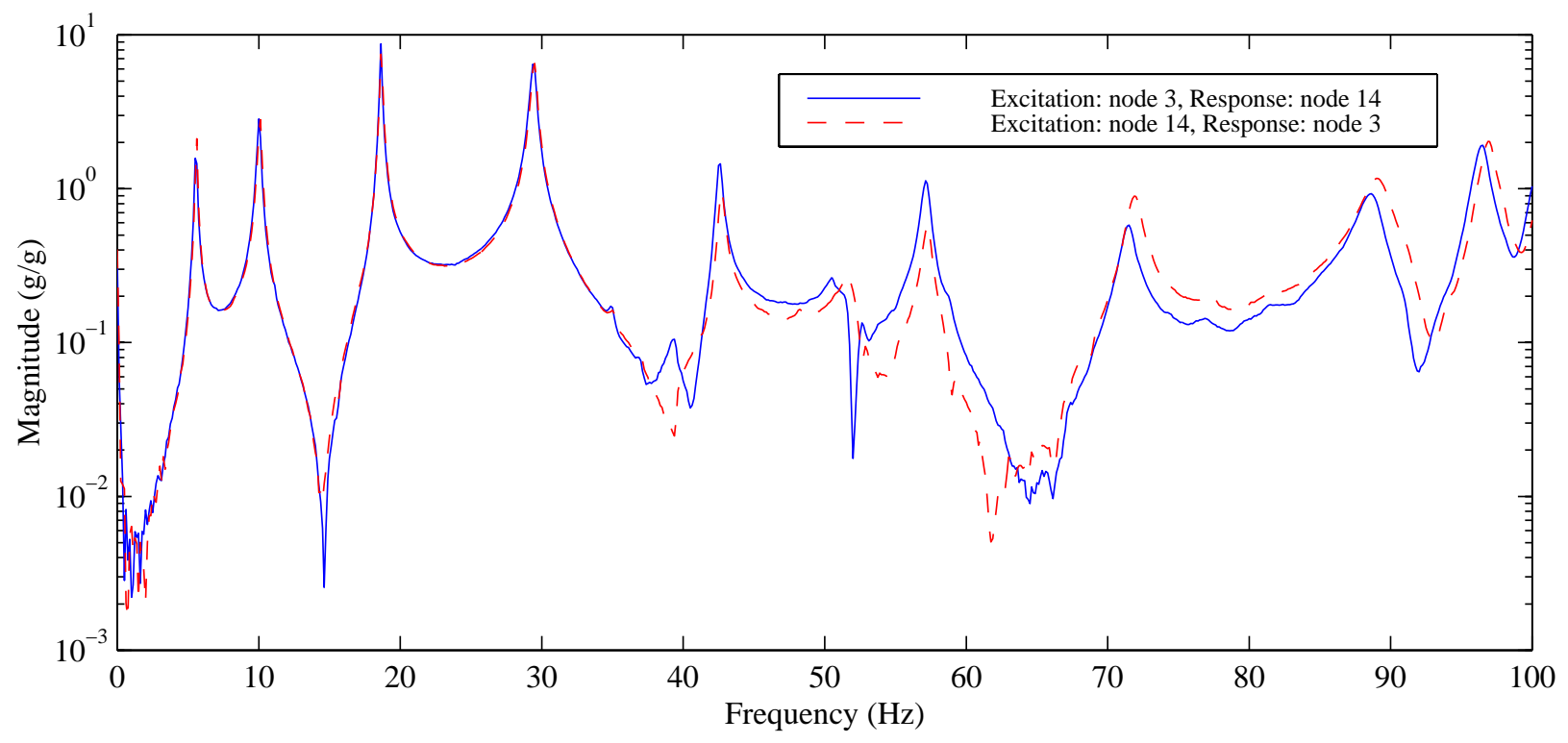

Figure 4: A reciprocity check of the grid-type bridge model 


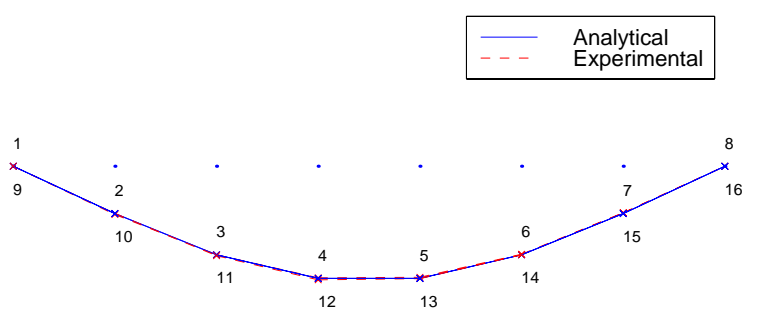

(a) Modal vector 1

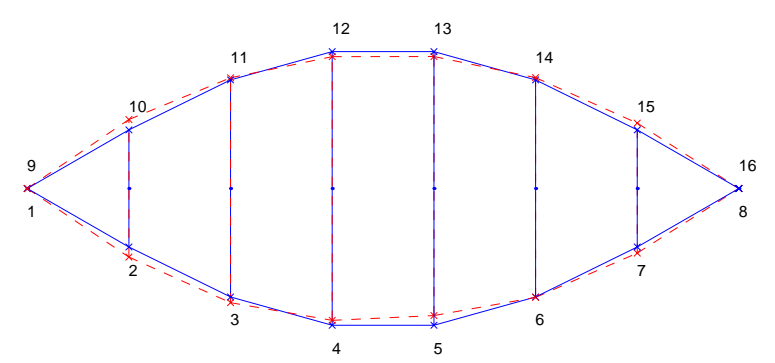

(b) Modal vector 2

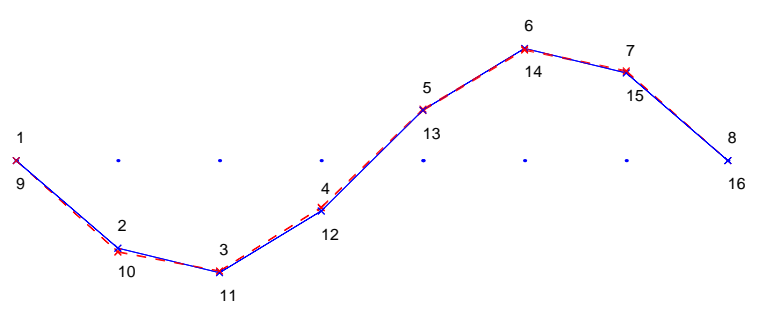

(c) Modal vector 3

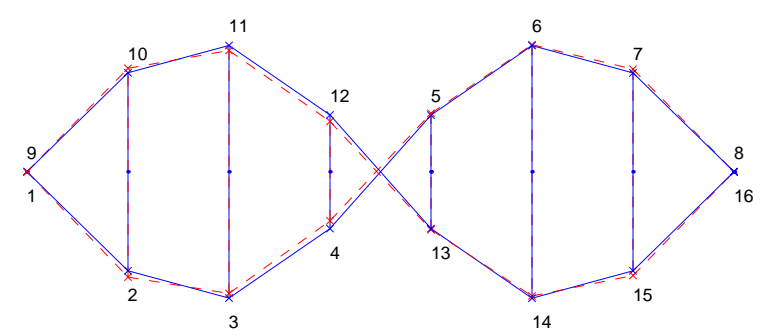

(d) Modal vector 4

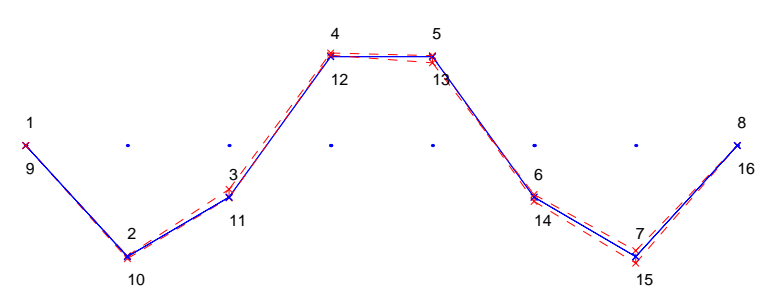

(e) Modal vector 5

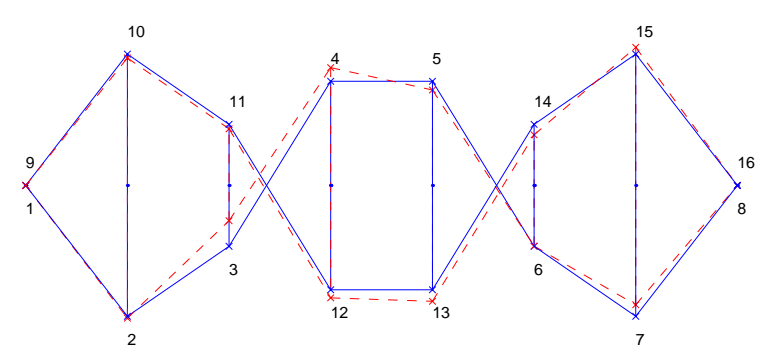

(f) Modal vector 6

Figure 5: Comparison of analytical and experimental modal vectors 


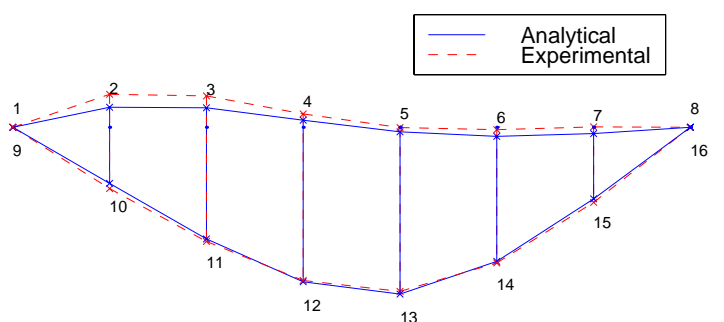

(a) Ritz vector 1

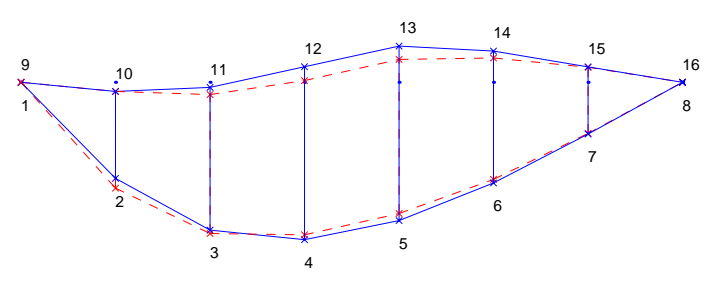

(b) Ritz vector 2

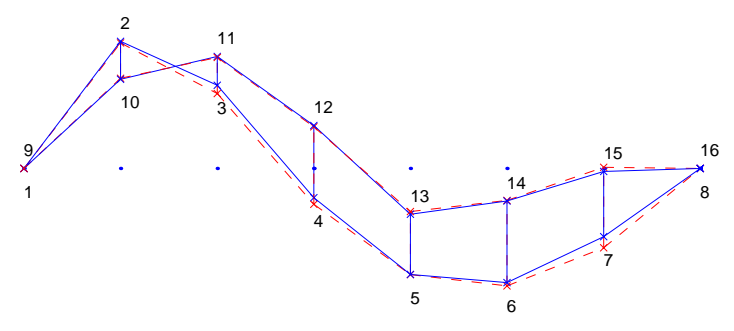

(c) Ritz vector 3

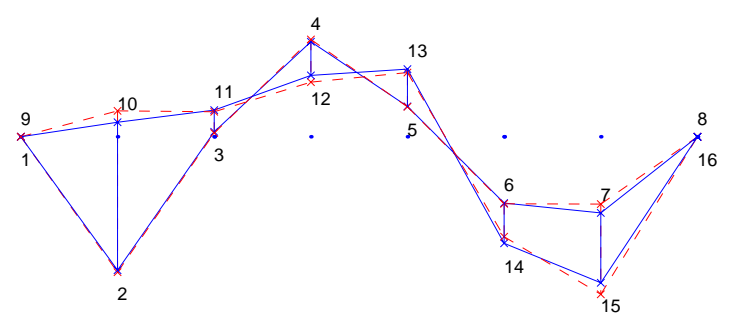

(d) Ritz vector 4

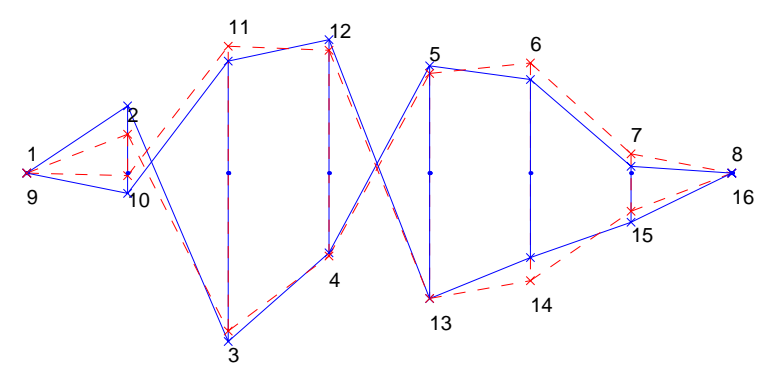

(e) Ritz vector 5

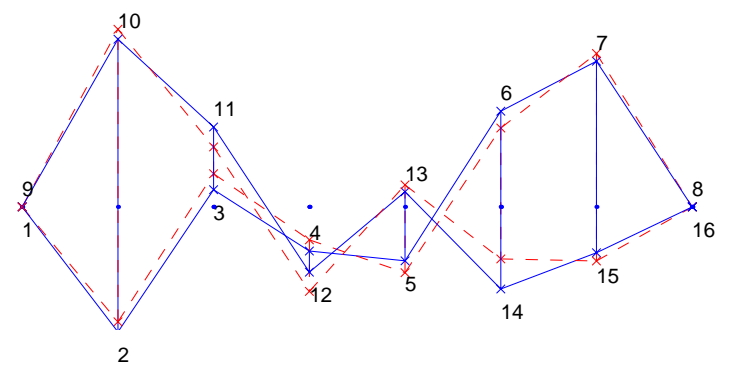

(f) Ritz vector 6

Figure 6: Comparison of analytical and experimental Ritz vectors (using a complete flexibility, $\left.\mathbf{G}=\mathbf{G}_{m}+\mathbf{G}_{r}\right)$ 


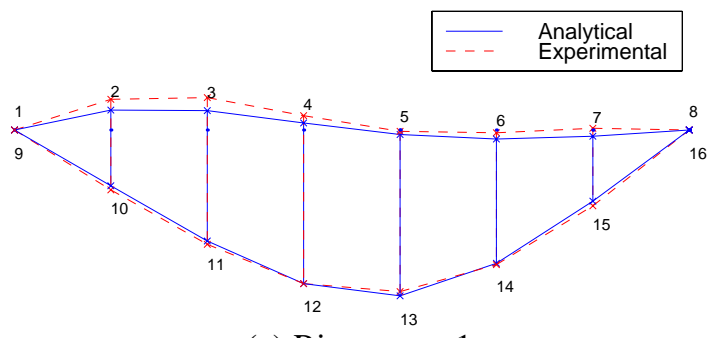

(a) Ritz vector 1

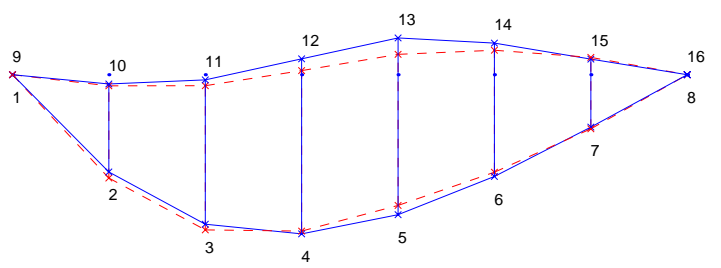

(b) Ritz vector 2

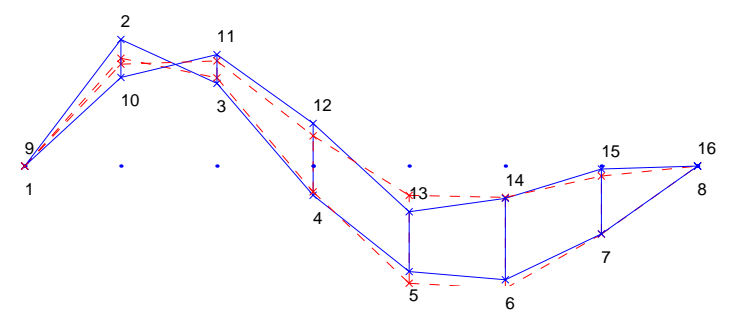

(c) Ritz vector 3

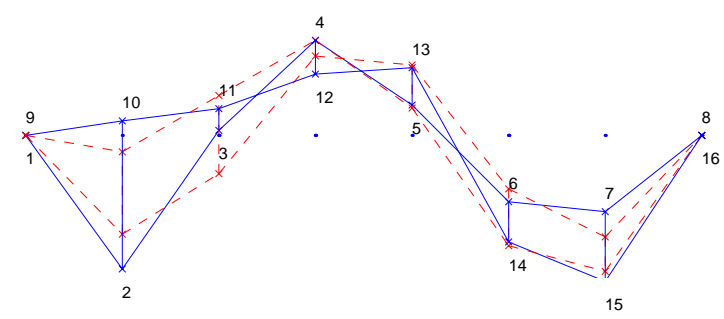

(d) Ritz vector 4

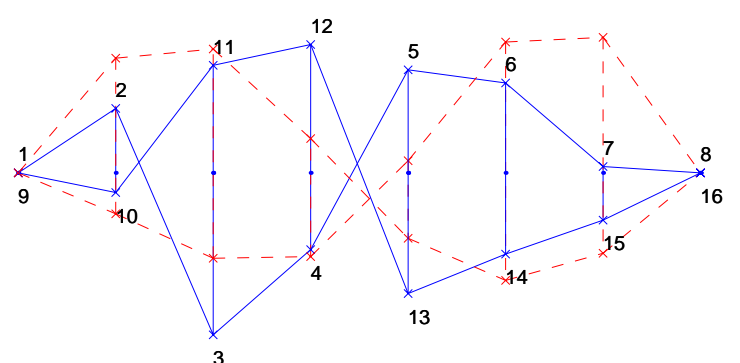

(e) Ritz vector 5

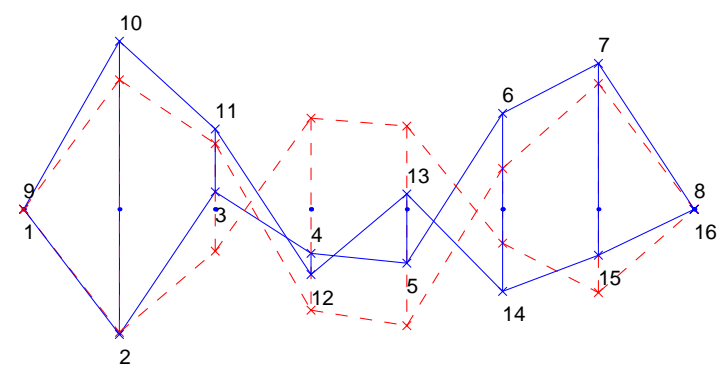

(f) Ritz vector 6

Figure 7: Comparison of analytical and experimental Ritz vectors (using only a modal flexibility, $\left.\mathbf{G}=\mathbf{G}_{m}\right)$ 
Table 1: Comparison of the analytical and experimental natural frequencies

\begin{tabular}{cccc}
\hline \multirow{2}{*}{ Mode } & \multicolumn{2}{c}{ Frequency $(\mathrm{Hz})$} & $\begin{array}{c}\text { Relative error* } \\
(\%)\end{array}$ \\
\hline \hline 1st Bending & Analytical & Experimental & 2.32 \\
1st Torsion & 5.4488 & 5.5780 & 0.88 \\
2nd Bending & 10.1494 & 10.0610 & 2.90 \\
2nd Torsion & 19.1841 & 18.6441 & 3.98 \\
3rd Bending & 30.6216 & 29.4503 & 2.39 \\
3rd Torsion & 41.6086 & 42.6292 & 3.98 \\
\hline
\end{tabular}

*error $=100 \times|\omega-\hat{\omega}| / \hat{\omega}: \omega$ and $\hat{\omega}$ are analytical and experimental frequencies. 
Table 2: MAC values between analytical and experimental Ritz vectors

\begin{tabular}{ccccccc}
\hline \multirow{G}{*}{} & \multicolumn{6}{c}{$i$ for $\operatorname{MAC}(i, i)$} \\
& 1 & 2 & 3 & 4 & 5 & 6 \\
\hline \hline $\mathbf{G}_{m}+\mathbf{G}_{r}$ & 0.9947 & 0.9930 & 0.9971 & 0.9956 & 0.9754 & 0.9720 \\
$\mathbf{G}_{m}$ & 0.9946 & 0.9930 & 0.9876 & 0.9099 & 0.3932 & 0.0211 \\
\hline \multirow{2}{*}{$\operatorname{MAC}(i, j)=\left(\mathbf{r}_{i}^{\mathrm{T}} \mathbf{M} \hat{\mathbf{r}}_{j}\right)^{2} /\left[\left(\mathbf{r}_{i}^{\mathrm{T}} \mathbf{M} \mathbf{r}_{j}\right)^{2}\left(\hat{\mathbf{r}}_{i}^{\mathrm{T}} \mathbf{M} \hat{\mathbf{r}}_{j}\right)^{2}\right], \mathbf{r}_{i}=$ analytical and $\hat{\mathbf{r}}_{j}=$ experimental. }
\end{tabular}


Table 3: MAC values for different load patterns (using the complete flexibility, $\mathbf{G}_{m}+\mathbf{G}_{r}$ )

\begin{tabular}{c|cccccc}
\hline \multirow{2}{*}{ Node } & \multicolumn{6}{|c}{$i$ for MAC $(i, i)$} \\
& 1 & 2 & 3 & 4 & 5 & 6 \\
\hline \hline 2 & 0.9944 & 0.9929 & 0.9980 & 0.9931 & 0.9888 & 0.9805 \\
3 & 0.9983 & 0.9964 & 0.9955 & 0.9945 & 0.9485 & 0.9657 \\
4 & 0.9994 & 0.9974 & 0.9966 & 0.9948 & 0.9857 & 0.9900 \\
5 & 0.9994 & 0.9966 & 0.9903 & 0.9907 & 0.9761 & 0.9656 \\
6 & 0.9989 & 0.9965 & 0.9967 & 0.9976 & 0.9755 & 0.9824 \\
7 & 0.9954 & 0.9952 & 0.9917 & 0.9800 & 0.9717 & 0.9731 \\
10 & 0.9937 & 0.9925 & 0.9950 & 0.9785 & 0.9697 & 0.9737 \\
11 & 0.9984 & 0.9956 & 0.9978 & 0.9978 & 0.9790 & 0.9849 \\
12 & 0.9995 & 0.9965 & 0.9932 & 0.9929 & 0.9825 & 0.9764 \\
13 & 0.9996 & 0.9970 & 0.9968 & 0.9970 & 0.9885 & 0.9877 \\
14 & 0.9993 & 0.9970 & 0.9951 & 0.9958 & 0.9542 & 0.9635 \\
15 & 0.9963 & 0.9953 & 0.9956 & 0.9935 & 0.9866 & 0.9819 \\
\hline *MAC $(i, j)=\left(\mathbf{r}_{i}^{\mathrm{T}} \mathbf{M} \hat{\mathbf{r}}_{j}\right)^{2} /\left[\left(\mathbf{r}_{i}^{\mathrm{T}} \mathbf{M} \mathbf{r}_{j}\right)^{2}\left(\hat{\mathbf{r}}_{i}^{\mathrm{T}} \mathbf{M} \hat{\mathbf{r}}_{j}\right)^{2}\right], \mathbf{r}_{i}=$ analytical and $\hat{\mathbf{r}}_{j}=$ \\
experimental.
\end{tabular}


Table 4: MAC values for different load patterns (using the modal flexibility, $\mathbf{G}_{m}$ )

\begin{tabular}{c|cccccc}
\hline \multirow{2}{*}{ Node } & \multicolumn{7}{|c}{$i$ for $\mathrm{MAC}(i, i)$} \\
& 1 & 2 & 3 & 4 & 5 & 6 \\
\hline 2 & 0.9944 & 0.9920 & 0.9913 & 0.9429 & 0.7541 & 0.3560 \\
3 & 0.9982 & 0.9958 & 0.9887 & 0.9502 & 0.4777 & 0.0848 \\
4 & 0.9993 & 0.9967 & 0.9678 & 0.9262 & 0.7802 & 0.3623 \\
5 & 0.9994 & 0.9958 & 0.9472 & 0.8818 & 0.6782 & 0.2588 \\
6 & 0.9989 & 0.9962 & 0.9909 & 0.9627 & 0.5722 & 0.1649 \\
7 & 0.9952 & 0.9933 & 0.9779 & 0.9019 & 0.6498 & 0.2654 \\
10 & 0.9938 & 0.9910 & 0.9827 & 0.9096 & 0.6753 & 0.2651 \\
11 & 0.9984 & 0.9952 & 0.9925 & 0.9621 & 0.5631 & 0.1578 \\
12 & 0.9995 & 0.9958 & 0.9569 & 0.8997 & 0.7208 & 0.2973 \\
13 & 0.9996 & 0.9964 & 0.9630 & 0.9139 & 0.7431 & 0.3377 \\
14 & 0.9992 & 0.9965 & 0.9895 & 0.9566 & 0.5213 & 0.1140 \\
15 & 0.9962 & 0.9938 & 0.9847 & 0.9306 & 0.7229 & 0.3424 \\
\hline
\end{tabular}

$* \operatorname{MAC}(i, j)=\left(\mathbf{r}_{i}^{\mathrm{T}} \mathbf{M} \hat{\mathbf{r}}_{j}\right)^{2} /\left[\left(\mathbf{r}_{i}^{\mathrm{T}} \mathbf{M} \mathbf{r}_{j}\right)^{2}\left(\hat{\mathbf{r}}_{i}^{\mathrm{T}} \mathbf{M} \hat{\mathbf{r}}_{j}\right)^{2}\right], \mathbf{r}_{i}=$ analytical and $\hat{\mathbf{r}}_{j}=$ experimental. 